\title{
Hydrogenated and deuterated iron clusters: Infrared spectra and density functional calculations
}

\author{
Mark B. Knickelbein and Geoffrey M. Koretsky \\ Chemistry Division, Argonne National Laboratory, Argonne, Illinois 60439 \\ Koblar A. Jackson \\ Department of Physics, Central Michigan University, Mt. Pleasant, Michigan 48859 \\ Mark R. Pederson \\ Complex Systems Theory Branch, Naval Research Laboratory, Washington, DC 20375 \\ Zoltan Hajnal \\ University-GH Paderborn Theoretische Physik, D-33095 Paderborn, Germany
}

(Received 26 August 1998; accepted 16 September 1998)

\begin{abstract}
Iron clusters react sequentially with hydrogen molecules to form multiply hydrogenated products. The increases in cluster ionization potential upon reaction verify that hydrogen chemisorbs dissociatively to form iron cluster-hydride complexes, $\mathrm{Fe}_{n} \mathrm{H}_{m}$. At low source temperatures, the cluster-hydride complexes take up additional hydrogen molecules which are shown to be physisorbed onto the underlying $\mathrm{Fe}_{n} \mathrm{H}_{m}$ complexes to form $\mathrm{Fe}_{n} \mathrm{H}_{m}\left(\mathrm{H}_{2}\right)_{p}$ species. The infrared spectra of $\mathrm{Fe}_{n} \mathrm{H}_{m}$ and $\mathrm{Fe}_{n} \mathrm{D}_{m}(n=9-20)$ were obtained by the photodissociation action spectroscopic method in which depletion of the $\mathrm{Fe}_{n} \mathrm{H}_{m}\left(\mathrm{H}_{2}\right)_{p}$ and $\mathrm{Fe}_{n} \mathrm{D}_{m}\left(\mathrm{D}_{2}\right)_{p}$ species was the signature of absorption. The spectra, recorded in the $885-1090 \mathrm{~cm}^{-1}$ region, consist of several overlapping bands, each approximately $20 \mathrm{~cm}^{-1}$ in width. The dissimilarity of each $\mathrm{Fe}_{n} \mathrm{H}_{m}\left(\mathrm{H}_{2}\right)_{p}$ spectrum with the corresponding $\mathrm{Fe}_{n} \mathrm{D}_{m}\left(\mathrm{D}_{2}\right)_{p}$ spectrum indicates that the carrier involves hydrogen and is not merely due to absorption by the underlying iron cluster. Density functional calculations were performed on model complexes, $\mathrm{Fe}_{13} \mathrm{H}_{14}$ and $\mathrm{Fe}_{13} \mathrm{D}_{14}$, the iron portion of which was assumed to have $T_{h}$ symmetry. The infrared-active vibrational frequencies involving hydrogen bending and deuterium stretching are predicted to lie within the experimental frequency range of the experiment, well removed from the skeletal modes of the underlying iron cluster. The complexity of the observed spectra as compared to simulations based on the assumed (high-symmetry) model imply that the experimentally produced complexes possess low symmetry. (C) 1998 American Institute of Physics. [S0021-9606(98)01148-9]
\end{abstract}

\section{INTRODUCTION}

One of the first indications that small clusters of transition metal atoms, $\mathbf{M}_{n}$, possess properties that are fundamentally different from the corresponding bulk metal emerged from gas-phase reactivity studies. In particular, the orders-ofmagnitude variation in reactivity with molecular hydrogen ${ }^{1-16}$ within a homologous series of clusters provided compelling evidence that these species were not simply small pieces of the bulk metal of varying size, but rather distinct species whose structure and properties varied significantly from one size to the next. While reaction kinetics studies of various $\mathrm{M}_{n}$ species have since been reported for a wide variety of molecular reactants, none have displayed the wide range in reactivity behavior exhibited by $\mathrm{M}_{n}+\mathrm{H}_{2}$ reactions. Consequently, hydrogen reactivity studies have continued to serve as important benchmarks in the development of structure-reactivity correlations in transition metal clusters $^{4,5,16,17}$ and have the additional attractiveness of being among the simplest classes of cluster-molecule reactions to be tractable by first-principles theoretical methods. ${ }^{18-22}$ One of the assumptions usually made in studies of $\mathrm{M}_{n}+\mathrm{H}_{2}$ reactions is that the observed products are the ultimate result of dissociative (rather than molecular) chemisorption on the surface of the cluster: $\mathrm{M}_{n}+\mathrm{H}_{2} \rightarrow \mathrm{M}_{n} \mathrm{HH}$. The most widely invoked argument in support of this mechanism is based on the known reaction pathways of hydrogen on the corresponding bulk transition metal surfaces, where dissociative chemisorption nearly universally occurs. ${ }^{23}$ The preponderance of experimental and theoretical evidence has been consistent with the dissociative chemisorption product channel for $\mathrm{M}_{n}$ $+\mathrm{H}_{2}$ reactions as well. Experimental evidence includes determinations of the energy required to desorb $\mathrm{H}_{2}$ from hydrogen covered clusters via multiphoton photodissociation ${ }^{8}$ and collision-induced dissociation, ${ }^{24}$ the observed increases of cluster ionization potential (IP) upon reaction with hydrogen, the apparent irreversibility of the $\mathrm{M}_{n}+\mathrm{H}_{2}$ reaction at (or exceeding) room temperature, ${ }^{3}$ and the detailed behavior of the $\mathrm{M}_{n}+\mathrm{H}_{2}$ reaction rate coefficient with temperature.

In this article, we present a combined experimental and theoretical study of hydrogen- and deuterium-covered iron clusters, $\mathrm{Fe}_{n} \mathrm{H}_{m}$ and $\mathrm{Fe}_{n} \mathrm{D}_{m}(n=9-20)$. A series of earlier studies ${ }^{2,5,6,9}$ have shown that among transition metal clusters studied to date, iron clusters display perhaps the most dramatic variation in their rate of reaction toward molecular hydrogen with size. It was further shown ${ }^{7}$ that $\mathrm{Fe}_{n}$ can react sequentially with hydrogen molecules, forming multiply hydrogenated species, and that under sufficiently high $\mathrm{H}_{2}$ concentrations, clusters saturated with a well-defined number of 
(what was presumed to be) hydrogen atoms are formed. It is these hydrogen-saturated iron clusters that are the subject of the present study. The infrared photodissociation spectra and supporting density functional-based calculations presented here provide a detailed characterization of metal clusterhydrogen vibrations in complexes formed as a result of dissociative chemisorption.

\section{EXPERIMENTAL METHODS}

The experimental apparatus and methods used to record infrared photodissociation spectra have been described in detail previously. ${ }^{25}$ Briefly, iron clusters, $\mathrm{Fe}_{n}$, were generated by pulsed laser vaporization from an isotopically enriched $(>99 \%){ }^{56} \mathrm{Fe}$ target. Cluster cooling and growth occurred in 20-25 Torr of helium carrier gas flowing within a $7.6 \mathrm{~cm} \times$ $0.32 \mathrm{~cm}$ (length $\times$ inner diameter) flow tube maintained at 70-300 K. The iron cluster-hydrogen (deuterium) complexes were formed by adding 5\%-15\% hydrogen (deuterium) to the helium flow stream $3 \mathrm{~cm}$ downstream of the target rod.

Following a mild supersonic expansion and skimming, the cluster beam entered into a detection chamber held at $\sim 10^{-6}$ Torr. Here the beam was crossed at $90^{\circ}$ by a collimated ArF (probe) laser $(h \nu=6.42 \mathrm{eV})$ which ionized the clusters for time-of-flight (TOF) mass analysis. On every other probe laser pulse, a line-tunable, pulsed ( $\Delta t=500 \mu \mathrm{s})$ carbon dioxide (pump) laser irradiated the molecular beam. The fractional depletion $D(\nu)$ of the cluster complexes was measured by operating the pump laser at one half the probe laser repetition rate and concurrently recording two TOF spectra: a reference TOF spectrum obtained with the $\mathrm{ArF}$ probe laser only, and a depletion TOF spectrum obtained using both pump and probe lasers. To cover the 990-1010 $\mathrm{cm}^{-1}$ and $885-920 \mathrm{~cm}^{-1}$ regions (normally not accessible with $\mathrm{CO}_{2}$ lasers), the laser was operated with ${ }^{13} \mathrm{CO}_{2}$ in the laser gas mixture. The relative pump laser fluence $F(\nu)$ was measured by reflecting a portion of the $\mathrm{CO}_{2}$ laser output into a pyroelectric energy detector.

For ionization potential bracketing studies, the frequency doubled output of a $\mathrm{XeCl}$ excimer laser-pumped dye laser was used in place of the ArF laser for photoionization.

\section{RESULTS AND DISCUSSION}

\section{A. Near-threshold photoionization studies}

Figure 1 shows TOF spectra of iron clusters in the range $\mathrm{Fe}_{9}$ to $\mathrm{Fe}_{20}$ recorded with varying hydrogen partial pressures within the cluster source. Under conditions of relatively low $\mathrm{H}_{2}$ partial pressure, the iron clusters react to various extents depending on their individual reactivities, leading to a congested TOF spectrum reflecting the distribution of partially hydrogenated clusters, as shown in Fig. 1(a). As the hydrogen content within the cluster source is increased to approximately $10 \%$, the mass spectrum simplifies as hydrogensaturated clusters are formed, shown in Fig. 1(b). The compositions of the hydrogen-saturated clusters observed in this work are in most cases identical to those reported previously by Richtsmeier et al. ${ }^{6}$ However for $\mathrm{Fe}_{10}, \mathrm{Fe}_{14}$, and $\mathrm{Fe}_{19-21}$, we observe a distribution of $\mathrm{Fe}_{n} \mathrm{H}_{m}$ species with

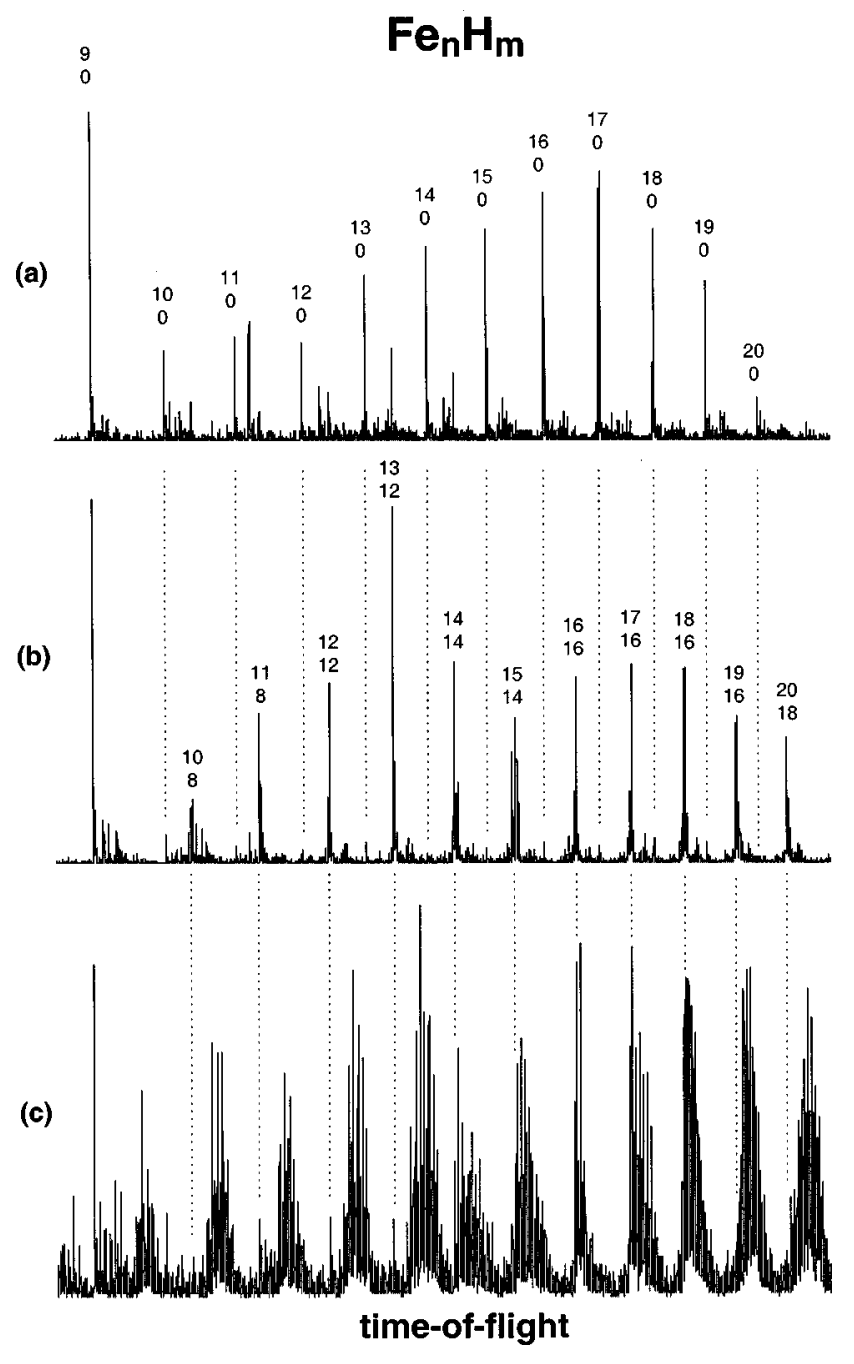

FIG. 1. Time-of-flight mass spectra of iron clusters at various flow tube temperatures and hydrogen content. (a) $\left[\mathrm{H}_{2}\right]:[\mathrm{He}]=1: 350, T=290 \mathrm{~K}$; (b) $\left[\mathrm{H}_{2}\right]:[\mathrm{He}]=1: 19, T=200 \mathrm{~K} ;\left[\mathrm{H}_{2}\right]:[\mathrm{He}]=1: 19, T=70 \mathrm{~K}$.

values of $m$ up to and including the saturation values that were reported in the previous work. For example, we observe $\mathrm{Fe}_{14} \mathrm{H}_{14-18}$, with $\mathrm{Fe}_{14} \mathrm{H}_{14}$ dominant, whereas Parks et al. reported only $\mathrm{Fe}_{14} \mathrm{H}_{18}$ as the saturated species. ${ }^{7}$ This result suggests incomplete saturation under the present experimental conditions. Increasing the hydrogen flow in order to fully saturate these clusters with hydrogen proved to be problematic, however, resulting in significantly decreased cluster intensity.

The narrow $\mathrm{Fe}_{n} \mathrm{H}_{m}$ product distribution shown in Fig. 1(b) was found to be largely independent of temperature between 150 and $300 \mathrm{~K}$, and will be referred to in the following discussion as high temperature complexes. At temperatures below $150 \mathrm{~K}$, the narrow mass distributions displayed by the high temperature complexes asymmetrically broadened to higher mass, resulting in a congested mass spectrum as shown in Fig. 1(c). Examination of the TOF mass spectrum clearly reveals that this broadening is due to uptake of additional hydrogen molecules. These complexes containing additional hydrogen will be referred to as low temperature complexes.

The use of $\mathrm{D}_{2}$ as the reactant yields the same overall 


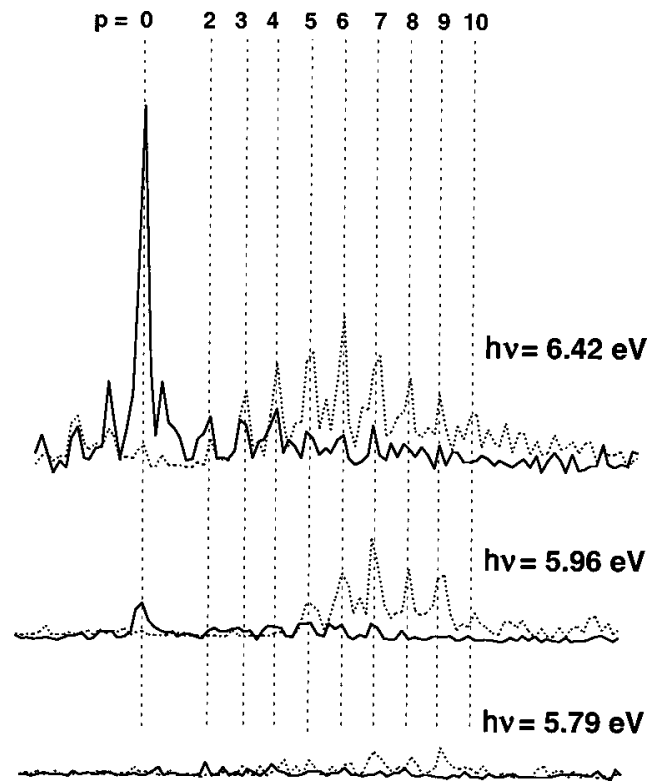

FIG. 2. Expanded view of the TOF mass spectrum of $\mathrm{Fe}_{13} \mathrm{H}_{12}\left(\mathrm{H}_{2}\right)_{p}$ recorded at various photoionization energies. Dashed spectrum: $\mathrm{CO}_{2}$ laser off. Solid spectrum: $\mathrm{CO}_{2}$ laser on, $\nu=1078.6 \mathrm{~cm}^{-1}$. Experimental conditions: $T_{\text {nozzle }}$ $=82 \mathrm{~K},\left[\mathrm{H}_{2}\right]:[\mathrm{He}]=1: 4.5$.

results as those presented above for $\mathrm{H}_{2}$ under otherwise similar flow and temperature conditions.

In order to determine the adsorbed state of hydrogen within the two types of complexes, we invoke the wellestablished correspondence between metal cluster ionization potential and metal surface work function, in particular how these properties vary with the presence of adsorbates. ${ }^{26-30} \mathrm{In}$ analogy to the increase in work function, $\Delta \phi$, usually observed to accompany the dissociative chemisorption of hydrogen on macroscopic transition metal surfaces (due to modification of the surface dipole), ${ }^{23,31}$ it is expected that iron clusters containing adsorbed atomic hydrogen will possess higher IPs than their bare cluster counterparts. Such IP increases have been measured in previous photoionization studies of hydrogenated clusters of nickel, ${ }^{32}$ iron, ${ }^{30}$ niobium, ${ }^{30}$ and vanadium ${ }^{30}$ atoms, and were cited as evidence supporting the dissociative chemisorption channel. Conversely, physisorption of molecular hydrogen is predicted to lead to an IP decrease due to charged-induced polarization effects, as discussed below.

Figure 2 shows details of a series of TOF spectra of $\mathrm{Fe}_{13}$-hydrogen complexes, recorded at three different photoionization energies. Under the low temperature and high $\mathrm{H}_{2}$ flow conditions used in acquiring this TOF spectrum, nearly all of the $\mathrm{Fe}_{13} \mathrm{H}_{12}$ (high temperature) complexes were converted to $\mathrm{Fe}_{13} \mathrm{H}_{12}\left(\mathrm{H}_{2}\right)_{p}$ complexes within the source. For these studies, the high temperature complexes were created by heating the low temperature complexes with the infrared pump laser prior to photoionization (vide infra). The TOF spectra of $\mathrm{Fe}_{13} \mathrm{H}_{12}$ clearly show that its IP is $0.2-0.4 \mathrm{eV}$ higher than that of bare $\mathrm{Fe}_{13}, 5.61 \mathrm{eV} .^{33}$ The other complexes in the $n=9-20$ range were similarly higher in IP than their bare cluster counterparts. The observed increase in IP upon hydrogenation of the clusters is in agreement with the observation of Zakin et al. for $\mathrm{Fe}_{9-10}$ and $\mathrm{Fe}_{23-25}$ (Ref. 30) and is taken as confirming evidence that the high temperature complexes are iron cluster-hydrides, correctly written as $\mathrm{Fe}_{n} \mathrm{H}_{m}$, formed as a result of dissociative chemisorption.

The TOF spectra shown in Fig. 2 exemplify a common behavior for each group of iron cluster-hydrogen complexes in the $n=9-20$ range: The $\mathrm{Fe}_{n} \mathrm{H}_{m}\left(\mathrm{H}_{2}\right)_{p}$ mass peaks are depleted by the pump laser, while those of the corresponding $\mathrm{Fe}_{n} \mathrm{H}_{m}$ are enhanced in intensity. Examination of Fig. 2 shows that the low temperature complexes posses slightly lower IPs than their corresponding $\mathrm{Fe}_{n} \mathrm{H}_{m}$ parents, demonstrating that the low temperature "layer" of hydrogen consists of physisorbed $\mathrm{H}_{2}$ molecules (vide infra). The laserinduced heating of the low temperature complexes thus leads to loss of a portion of the physisorbed $\mathrm{H}_{2}$ from the low temperature complexes, with a corresponding enhancement of the high temperature complexes:

$$
\mathrm{Fe}_{n} \mathrm{H}_{m}\left(\mathrm{H}_{2}\right)_{p} \rightarrow \mathrm{Fe}_{n} \mathrm{H}_{m}\left(\mathrm{H}_{2}\right)_{p-q}+q \mathrm{H}_{2}
$$

This behavior is consistent with a strongly bound (chemisorbed) first layer of hydrogen atoms and a weakly bound (physisorbed) second layer of molecular hydrogen that adsorbs only at low temperatures. That the low temperature layer of hydrogen is physisorbed rather than chemisorbed is further supported by a classical electrostatic model which treats metal cluster complexes containing physisorbed atoms or molecules as spherical metal droplets with polarizable point bodies at their surfaces. ${ }^{28,29}$ This model predicts that a complex consisting of a metal cluster having physisorbed atoms (or molecules) on its surface will possess a lower IP than that of the underlying cluster, due to the charge-induced dipole energy of the polarizable adsorbate. The predictions of this model have been verified in threshold photoionization studies of metal cluster rare gas complexes, including $\mathrm{Nb}_{n} \mathrm{Ar}_{m}$ and $\mathrm{Ag}_{n} \mathrm{Kr}_{m} \cdot{ }^{28,34}$ This prediction is in agreement with the observation that the low temperature complexes have lower IPs than the higher temperature complexes, as shown in Fig. 2. A similar IP-lowering effect, also attributed to physisorbed molecular hydrogen, has been observed on fully hydrogenated nickel clusters formed at low temperatures. ${ }^{12}$

\section{B. Infrared photodissociation spectra}

The infrared absorption spectra of the $\mathrm{Fe}_{n} \mathrm{H}_{m}\left(\mathrm{H}_{2}\right)_{p}$ and $\mathrm{Fe}_{n} \mathrm{D}_{m}\left(\mathrm{D}_{2}\right)_{p}$ complexes were measured by depletion of the corresponding TOF mass peaks. As shown in Fig. 3, the depletion $D(\nu)$ of the $\mathrm{Fe}_{n} \mathrm{H}_{m}\left(\mathrm{H}_{2}\right)_{p}$ complexes increases monotonically with pump laser fluence $F(\nu)$, displaying the sigmoidal fluence saturation behavior expected for a simple first order depletion kinetics expression given by

$$
D(\nu)=D_{\max }\left(1-e^{-\sigma(\nu) F(\nu)}\right),
$$

where $\sigma(\nu)$ is the effective cross section. In this experiment, $D_{\max }$, the observed depletion at the limit of infinite pump fluence, was determined to be approximately 0.8 . As shown in Fig. 3, the fluence variation of the measured depletion in the $\mathrm{Fe}_{n} \mathrm{H}_{m}\left(\mathrm{H}_{2}\right)_{p}$ complexes is in accord with the first order model given by Eq. (2). Accordingly, effective absorption 


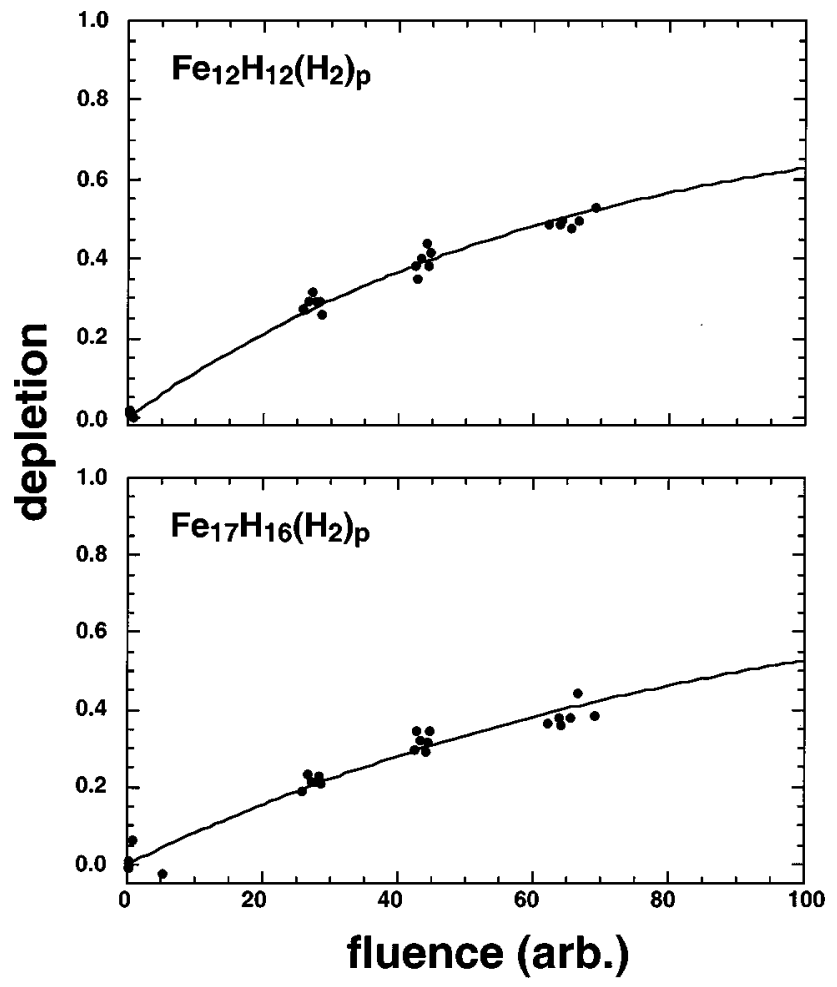

FIG. 3. Depletion vs pump laser fluence for representative $\mathrm{Fe}_{12} \mathrm{H}_{12}\left(\mathrm{H}_{2}\right)_{p}$ and $\mathrm{Fe}_{17} \mathrm{H}_{16}\left(\mathrm{H}_{2}\right)_{p}$ complexes. The observed depletion of the integrated mass peak intensities is for a range of $p$, typically from $p=2$ to $p=p_{\max }$, the highest mass low temperature complexes observed for a given $n$. The solid curves are fits to Eq. (2), with $D_{\max }=0.8$.

cross sections $\sigma(\nu)$ were obtained via Eq. (2) from $D(\nu)$ and $F(\nu)$ measured at discrete frequencies $\nu$ throughout the $885-1090 \mathrm{~cm}^{-1}$ region. ${ }^{35}$

The photodissociation action spectra of $\mathrm{Fe}_{n} \mathrm{H}_{m}\left(\mathrm{H}_{2}\right)_{p}$ and $\mathrm{Fe}_{n} \mathrm{D}_{m}\left(\mathrm{D}_{2}\right)_{p}(n=9-20)$ are shown in Figs. 4-6. One general feature is immediately evident: The spectra are complex, each consisting of many overlapping bands. The position and intensity of the bands was put on a more quantitative footing through least-squares fits of the spectral profiles, the general form of which was assumed to consist of a sum of Lorentzian functions with $20 \mathrm{~cm}^{-1}$ full width at half maximum (FWHM). The spectrum of each $\mathrm{Fe}_{n} \mathrm{H}_{m}\left(\mathrm{H}_{2}\right)_{p}$ complex clearly differs from that of the corresponding $\mathrm{Fe}_{n} \mathrm{D}_{m}\left(\mathrm{D}_{2}\right)_{p}$ species, verifying that the observed bands are due to vibrations involving hydrogen and deuterium and are not due to absorption by the underlying iron cluster. Notably, there were no obvious similarities in the spectra among the $\mathrm{Fe}_{n} \mathrm{H}_{m}\left(\mathrm{H}_{2}\right)_{p}$ complexes nor among the $\mathrm{Fe}_{n} \mathrm{D}_{m}\left(\mathrm{D}_{2}\right)_{p}$ species. Because of the possible existence of multiple isomers for a given $n$, the observed spectra may be convolutions, with each structure contributing intensity to the observed spectrum with a weight proportional to its relative population in the beam. Similarly, the distribution of $\mathrm{Fe}_{n} \mathrm{H}_{m}$ produced for $n$ $=10,14,19$, and 20 under the experimental conditions used will result in spectra that are weighted averages.

High resolution electron energy loss spectra of dissociatively chemisorbed $\mathrm{H}$ atoms on $\mathrm{Fe}(110)$ reveal features at 1060 and $880 \mathrm{~cm}^{-1}$, assigned to hydride stretching and bending motion, respectively. ${ }^{36}$ The observation of depletion features for the $\mathrm{Fe}_{n} \mathrm{H}_{m}\left(\mathrm{H}_{2}\right)_{p}$ complexes in this same general region of the spectrum would suggest that these features are due to iron cluster-hydride vibrations, i.e., that the IR-active chromophores within the $\mathrm{Fe}_{n} \mathrm{H}_{m}\left(\mathrm{H}_{2}\right)_{p}$ complexes are the underlying $\mathrm{Fe}_{n} \mathrm{H}_{m}$ species. Although no surface vibrational spectroscopy studies of chemisorbed deuterium on iron surfaces have (to our knowledge) been reported, the corresponding perpendicular $\mathrm{Fe}-\mathrm{D}$ stretching fundamental is expected to be centered near $750 \mathrm{~cm}^{-1}$ (based on the harmonic approximation), with the bending modes somewhat lower in frequency. Thus, the presence of absorption features in the $885-1090 \mathrm{~cm}^{-1}$ region for $\mathrm{Fe}_{n} \mathrm{D}_{m}\left(\mathrm{D}_{2}\right)_{p}$ is not expected based on extrapolation of the $\mathrm{H} / \mathrm{Fe}(110)$ chemisorption results. The complexity and variety displayed in the spectra of both $\mathrm{Fe}_{n} \mathrm{H}_{m}\left(\mathrm{H}_{2}\right)_{p}$ and $\mathrm{Fe}_{n} \mathrm{D}_{m}\left(\mathrm{D}_{2}\right)_{p}$ complexes rule out any interpretation adapted from studies on macroscopic surfaces. Rather, a detailed molecular model is required.

To further investigate the vibrational modes of $\mathrm{Fe}_{n} \mathrm{H}_{m}$ and $\mathrm{Fe}_{n} \mathrm{D}_{m}$ clusters, we carried out first-principles calculations based on the density functional theory (DFT) ${ }^{37,38}$ in the local spin density approximation (LSDA). DFT calculations are known to describe structural and vibrational properties very accurately. ${ }^{39,40}$ Several DFT calculations have been done on bare Fe clusters, including up to seven atoms, ${ }^{41-44}$ but we are not aware of calculations on hydrogenated clusters. For the $\mathrm{Fe}_{n} \mathrm{H}_{m}$ clusters studied here, precise structures are not known and the size of these clusters prevents an extensive search of cluster configurations using firstprinciples calculations to find the minimum energy structures. Lacking definitive structures, we opted to study the $\mathrm{Fe}_{13} \mathrm{H}_{14}$ model shown in Fig. 7. This model features an iron cluster core consisting of a central atom surrounded by 12 neighbors in approximate icosahedral symmetry. Among possible high-symmetry isomers, Dunlap found the icosahedral $\mathrm{Fe}_{13}$ cluster lowest in energy in a density functional calculation. ${ }^{45}$ Six of the $\mathrm{H}$ atoms are twofold coordinated, bridging surface $\mathrm{Fe}-\mathrm{Fe}$ bonds. The remaining eight $\mathrm{H}$ atoms are threefold coordinated. We refer to these below as bridge atoms and face atoms, respectively. With the $\mathrm{H}$ atoms, the model in Fig. 7 has $D_{2 h}$ symmetry. Experiments suggest that the saturated coverage for $\mathrm{Fe}_{13}$ is 12 atoms, rather than the 14 atoms shown in Fig. 7. However, the model in Fig. 7 has the significant computational advantage of being highly symmetric. Since we do not know the detailed structure for any of the clusters observed here experimentally, and can thus only expect qualitative agreement with the experiments, computational expediency was the crucial consideration in adopting the model shown in Fig. 7. We comment below on possible effects this choice may have on our results.

The calculations were done using the Naval Research Laboratory Molecular Orbital Library (NRLMOL) densityfunctional codes. ${ }^{46,47}$ This package features Gaussian-orbital basis sets and a novel numerical integration scheme ${ }^{47}$ that produces highly accurate total energies and atomic forces. A conjugate gradient algorithm is used to perform local geometry optimizations; vibrational frequencies are computed in the harmonic approximation as described previously. ${ }^{48}$ The all-electron calculations use large basis sets featuring 20 even-tempered Gaussians contracted to seven s-type, five $p$-type, and four $d$-type orbitals for the Fe atoms, and six 

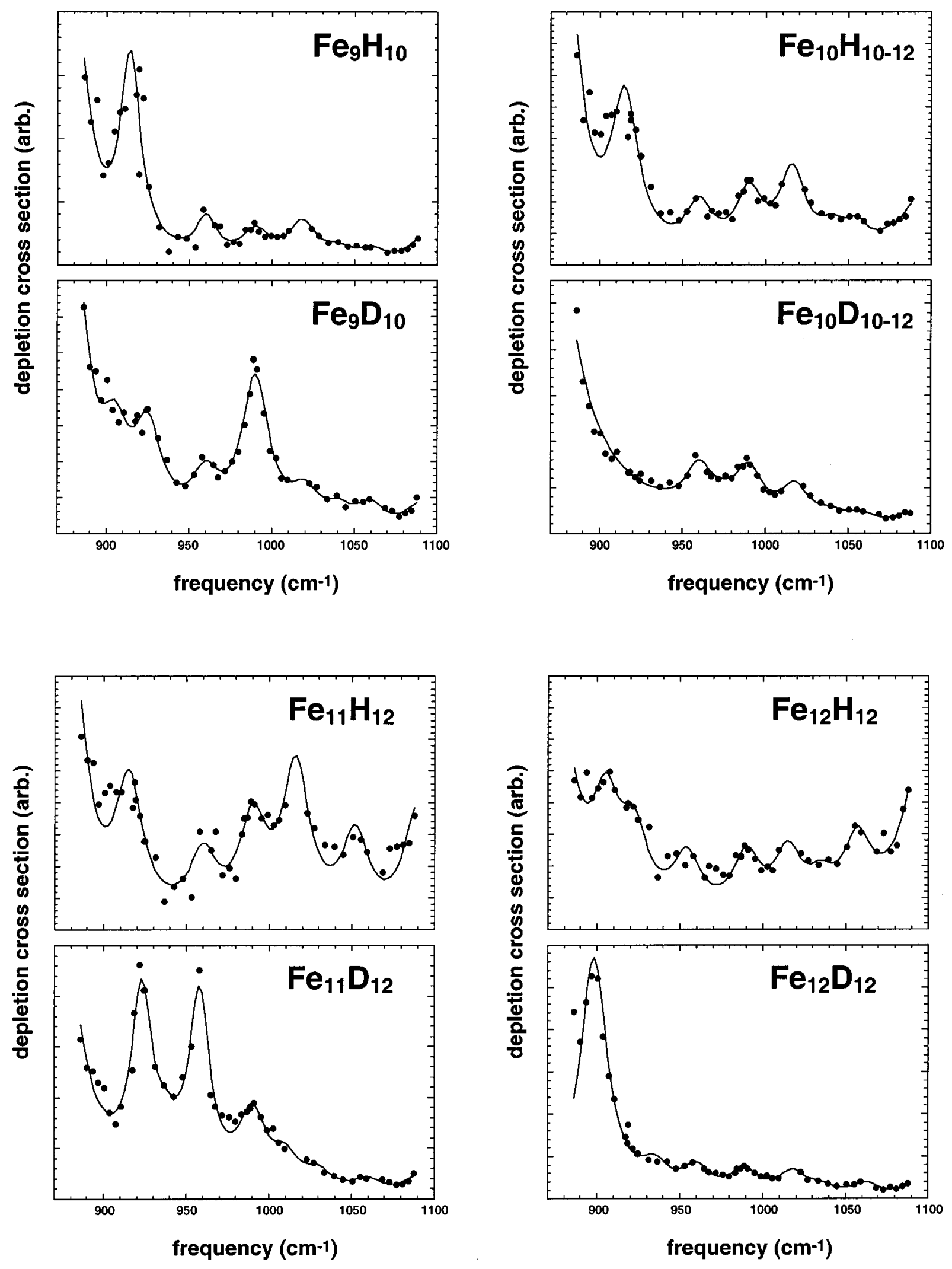

FIG. 4. Photodissociation action spectra of $\mathrm{Fe}_{n} \mathrm{H}_{m}\left(\mathrm{H}_{2}\right)_{p}$ and $\mathrm{Fe}_{n} \mathrm{D}_{m}\left(\mathrm{D}_{2}\right)_{p}$ for $n=9-12$.

Gaussians contracted to four $s$-type, three $p$-type, and one $d$-type for the $\mathrm{H}$ atoms. The equilibrium spin state for this model was found to be $S=7$ (i.e., 14 unpaired spins.)

We began by relaxing the cluster shown in Fig. 7 to a local minimum energy structure, maintaining the constraint of $T_{h}$ symmetry. After relaxation, there were two indepen- dent $\mathrm{Fe}-\mathrm{Fe}$ bond lengths on the cluster surface, $2.32 \AA$ for the bonds bridged by $\mathrm{H}$ atoms and $2.38 \AA$ for the others. The bond from the central $\mathrm{Fe}$ to the surface atoms was $2.25 \AA$. The $\mathrm{Fe}-\mathrm{H}$ bond was $1.66 \AA$ for the bridge $\mathrm{Hs}$ and $1.76 \AA$ for the face H's. We next computed the full vibrational spectrum for this structure. We found six zero frequencies and no 

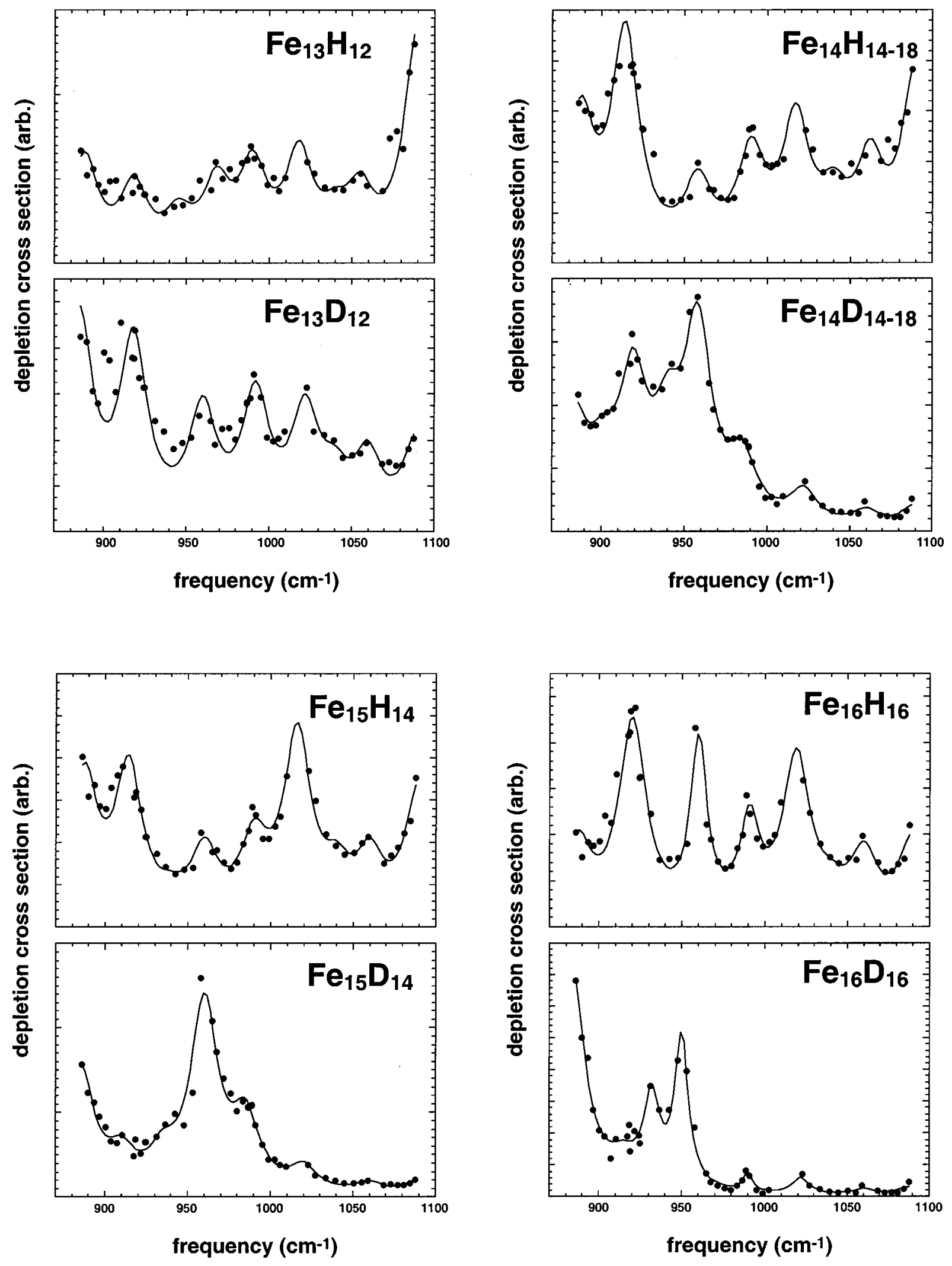

FIG. 5. Photodissociation action spectra for $\mathrm{Fe}_{n} \mathrm{H}_{m}\left(\mathrm{H}_{2}\right)_{p}$ and $\mathrm{Fe}_{n} \mathrm{D}_{m}\left(\mathrm{D}_{2}\right)_{p}$ for $n=13-16$.

imaginary frequencies, demonstrating that the cluster is locally stable. The calculated cluster vibrational density of states is shown in Fig. 8. The plot was made using a $10 \mathrm{~cm}^{-1}$ Lorentzian broadening of the discrete vibrational frequencies. Five bands of states can be seen in Fig. 8. The band between 0 and $400 \mathrm{~cm}^{-1}$ corresponds to vibrations of the $\mathrm{Fe}$ cluster core; the band between 800 and $1000 \mathrm{~cm}^{-1}$ involves bending modes of the face atoms; a narrow band between 1200 and $1250 \mathrm{~cm}^{-1}$ corresponds to bending modes of the bridge atoms; from 1250 to $1350 \mathrm{~cm}^{-1}$ are stretching modes of the face atoms; and from $1450-1500 \mathrm{~cm}^{-1}$ are stretching modes of the bridge atoms. The bending modes can be de- 

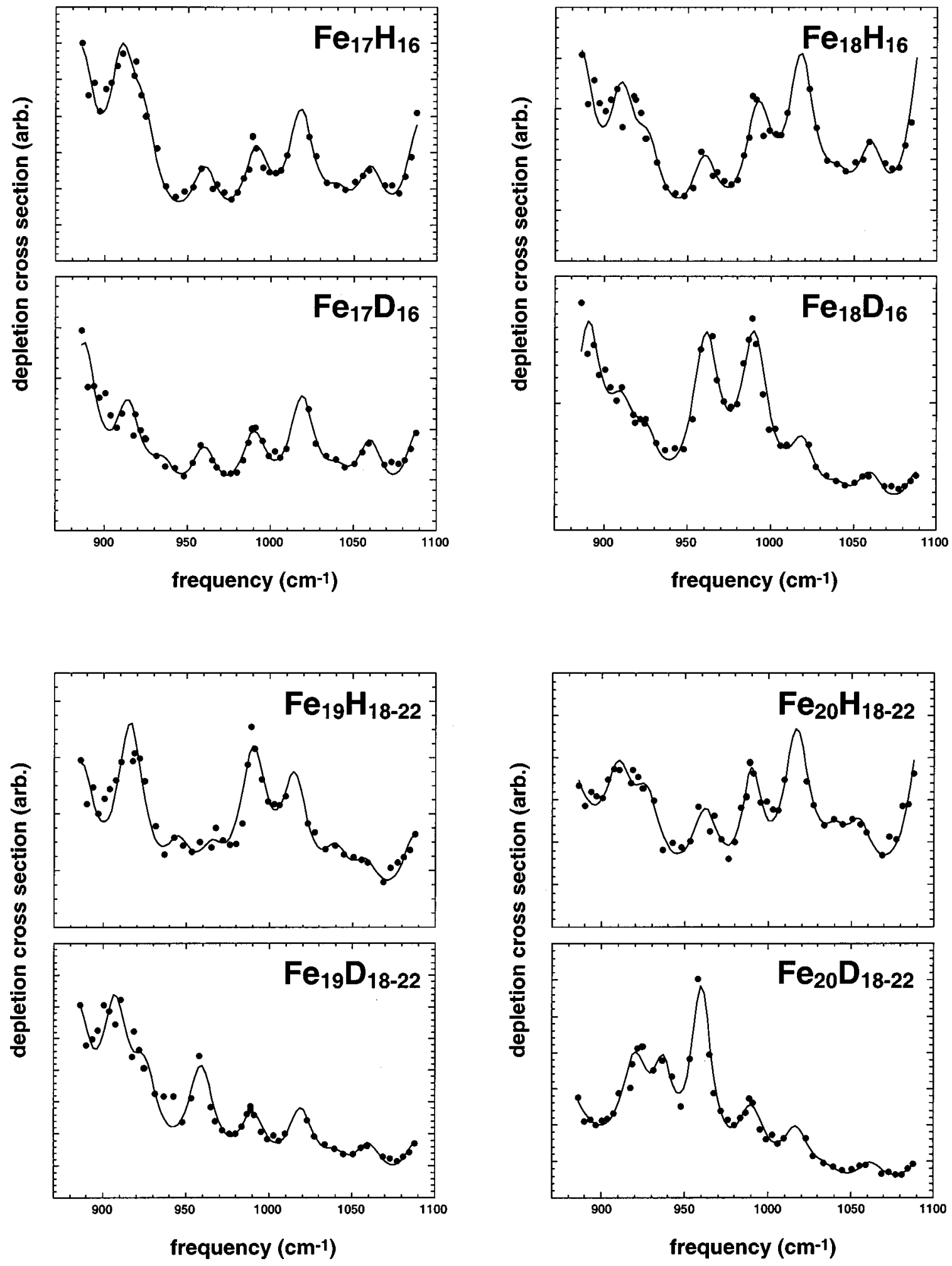

FIG. 6. Photodissociation action spectra of $\mathrm{Fe}_{n} \mathrm{H}_{m}\left(\mathrm{H}_{2}\right)_{p}$ and $\mathrm{Fe}_{n} \mathrm{D}_{m}\left(\mathrm{D}_{2}\right)_{p}$ for $n=17-20$.

scribed as motions of the H's that are parallel to the local cluster surface, while the stretching modes involve motion perpendicular to the surface. The splitting of the bands corresponding to the bridge and face atoms reflects the differences in the respective bonding environments.

In Fig. 9 we show a weighted density of states plot for the bands corresponding to $\mathrm{H}$ motion. The weights used correspond to the sum of the squares of the eigenvector compo- nents for each mode for the bridge and face atoms, respectively. The segregation of the bands is clearly seen. A similar plot for the spectrum of $\mathrm{Fe}_{13} \mathrm{D}_{14}$ can be generated by substituting the deuterium mass for the hydrogen mass in constructing the dynamical matrix. The isotope shift of the modes is found to be essentially $1 / \sqrt{2}$, consistent with the fact that these modes involve almost exclusively $\mathrm{H}$ motion. Interestingly, the bands corresponding to stretch modes are 


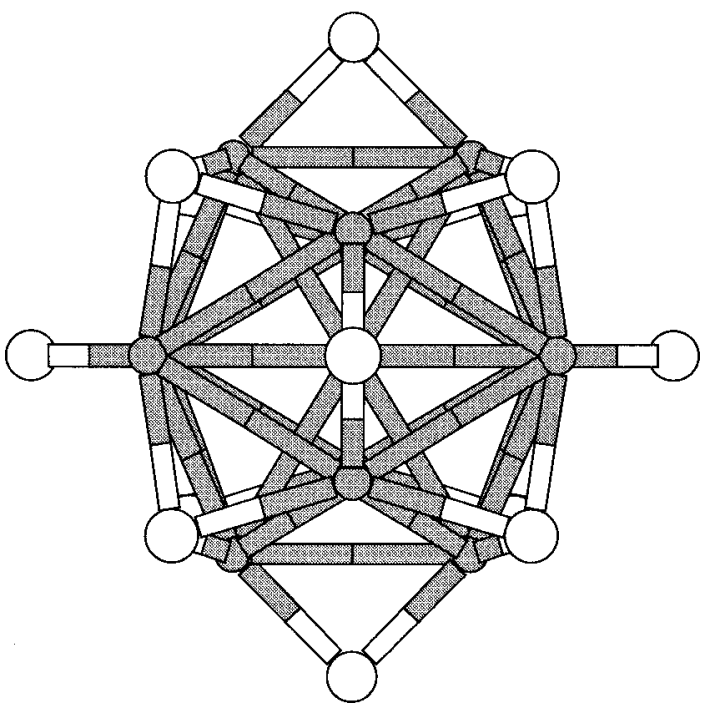

FIG. 7. Structure of $\mathrm{Fe}_{13} \mathrm{H}_{14}$ assumed for the LSDA calculations (see the text).

shifted downward to the ranges $885-955 \mathrm{~cm}^{-1}$ and $1025-$ $1061 \mathrm{~cm}^{-1}$, respectively, for the face and bridge atoms. Thus, both $\mathrm{Fe}_{13} \mathrm{H}_{14}$ and $\mathrm{Fe}_{13} \mathrm{D}_{14}$ have significant nonzero density of states in the region accessible to the pump laser, but the nature of the modes found in that region is different in the two cases. For $\mathrm{Fe}_{13} \mathrm{H}_{14}$, bending modes predominate, while for $\mathrm{Fe}_{13} \mathrm{D}_{14}$, stretching modes contribute most to the density of states.

In Fig. 10 we indicate the IR strengths of the vibrational fundamentals of both $\mathrm{Fe}_{13} \mathrm{H}_{14}$ and $\mathrm{Fe}_{13} \mathrm{D}_{14}$, calculated from the change in the cluster dipole moment with respect to the corresponding normal mode coordinate. ${ }^{48}$ For $\mathrm{Fe}_{13} \mathrm{H}_{14}$, it can be seen that the IR strength is concentrated in only a few of the modes, those at 850,1300 , and $1460 \mathrm{~cm}^{-1}$. The mode at $1205 \mathrm{~cm}^{-1}$ also has some intensity. For $\mathrm{Fe}_{13} \mathrm{D}_{14}$, the predominant IR-active modes lie at 605,925 , and $1040 \mathrm{~cm}^{-1}$.

The results shown in Figs. 8 and 9 are in good qualitative agreement with the measured photodissociation results for both the hydrogenated and deuterated clusters. They clearly show vibrational densities of states corresponding to $\mathrm{H}$-atom/D-atom modes in the region probed by the experi-

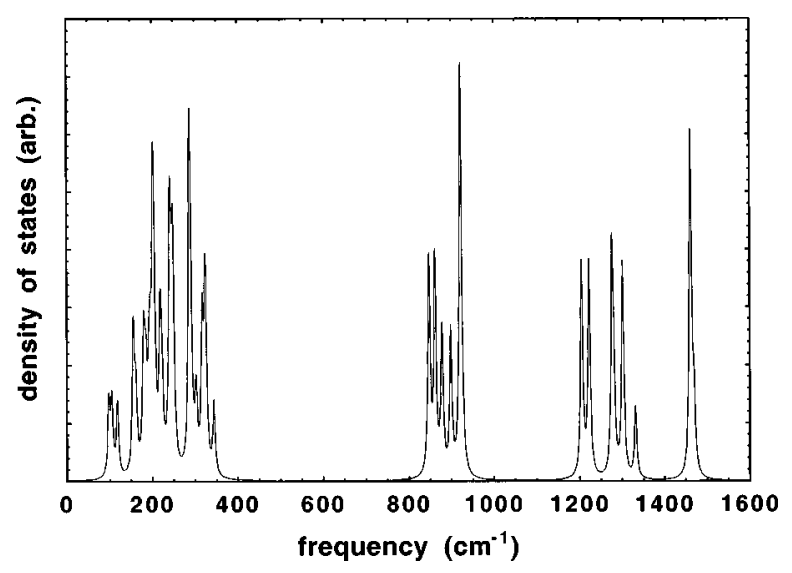

FIG. 8. Total vibrational density of states for $\mathrm{Fe}_{13} \mathrm{H}_{14}$.

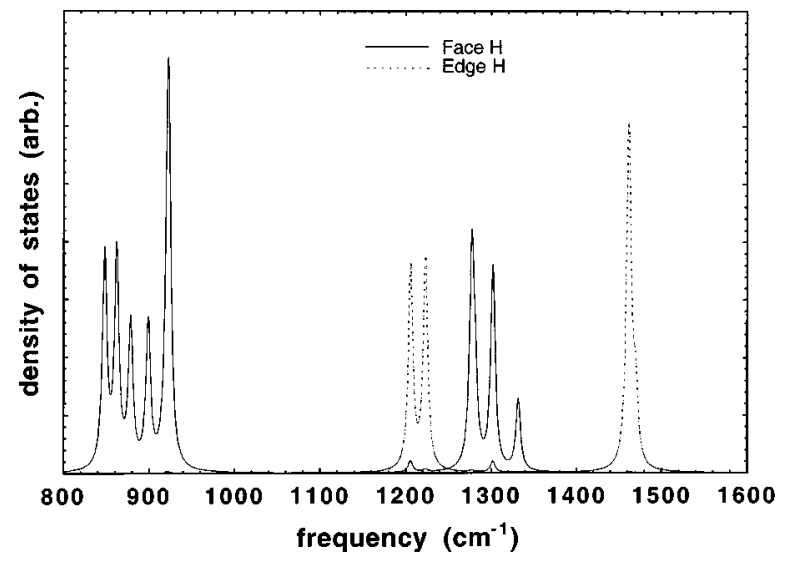

FIG. 9. Weighted density of states for edge- and face-bound $\mathrm{H}$ in $\mathrm{Fe}_{13} \mathrm{H}_{14}$.

ment. Modes associated with the Fe core are well-removed from the frequency window of the pump laser. An obvious difference between the calculated and measured results is the fact that the IR strength shown in Fig. 10 is concentrated in only a few modes, while the experiments suggest that the strength is spread more evenly across many vibrational bands. The high symmetry of our cluster model is likely to be responsible for the concentration of the IR strength, since only vibrational modes of appropriate symmetry can be IR active. The actual cluster geometries are likely to be less symmetric. For example, the saturation coverage for $\mathrm{Fe}_{13}$ is $12 \mathrm{H}$ atoms. While highly symmetric octahedral and icosahedral (as in Fig. 7) models can be constructed for $\mathrm{Fe}_{13}, 12 \mathrm{H}$ atoms cannot be placed on bridge or face sites in a way that preserves the symmetry. Lowering the symmetry of the cluster would have the effect of splitting degenerate modes and distributing IR strength more broadly across the vibrational bands. The net effect would be a somewhat wider distribution of bands with a more dispersed IR activity. For the limiting case where the structure of a given $\mathrm{Fe}_{n} \mathrm{H}_{m}$ species possesses no symmetry elements $\left(C_{1}\right)$, there will be $3 m$ distinct normal modes involving (primarily) hydrogen motion. As noted above, the presence of multiple isomers for a given $n$ could also be a contributing factor to the congested spectra.

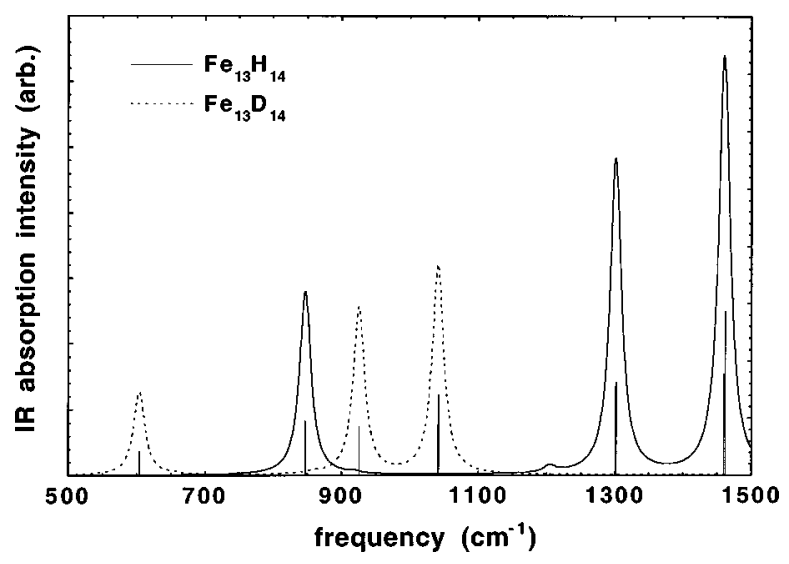

FIG. 10. Calculated IR spectra for $\mathrm{Fe}_{13} \mathrm{H}_{14}$ and $\mathrm{Fe}_{13} \mathrm{D}_{14}$. The Lorentzian FWHM was assumed to be $20 \mathrm{~cm}^{-1}$. 
Another difference between the calculated and observed bands is that the calculated bending modes are somewhat softer than might be expected from experiment. The spectra in Figs. 4-6 suggest that there are IR-active modes below $885 \mathrm{~cm}^{-1}$ where the bulk of the calculated vibrational density of states lies, but they also suggest that the band extends up to around $1100 \mathrm{~cm}^{-1}$, whereas the calculated band extends only to around $1000 \mathrm{~cm}^{-1}$. As argued above, lowering the symmetry of the model may broaden the calculated spectrum somewhat; however, another effect that may play a role is the use of $14 \mathrm{H}$ atoms in our model, as compared to the 12 $\mathrm{H}$ atoms present in the saturated clusters observed in experiments. Removing two $\mathrm{H}$ atoms from $\mathrm{Fe}_{13} \mathrm{H}_{14}$ would leave the remaining $\mathrm{H}$ atoms more strongly bound to the $\mathrm{Fe}$ core and thereby stiffen the calculated modes. As a test of this idea, we computed the vibrational modes for a smaller model, $\mathrm{Fe}_{4} \mathrm{H}_{6}$. The Fe core was tetrahedral, and the $\mathrm{H}$ atoms were placed on the six bridge sites. The bending modes for this system fell in the range of $940-1000 \mathrm{~cm}^{-1}$. Removing two of the $\mathrm{H}$ atoms and recalculating the vibrational modes, we found the bending modes of $\mathrm{Fe}_{4} \mathrm{H}_{4}$ to fall in the range of $1045-1060 \mathrm{~cm}^{-1}$.

\section{CONCLUSIONS}

The work reported here represents the first direct probe of the vibrational modes of atoms chemisorbed to Fe clusters. The measurements indicate broad IR absorption of the hydride clusters across the range of the $\mathrm{CO}_{2}$ pump laser, from $885-1090 \mathrm{~cm}^{-1}$, for both hydrogenated and deuterated clusters. First-principles calculations on a model $\mathrm{Fe}_{13} \mathrm{H}_{14}$ cluster are in good qualitative agreement with the measurements, showing broad $\mathrm{Fe}-\mathrm{H}$ and $\mathrm{Fe}-\mathrm{D}$ vibrational bands in the pump laser window and confirming that the modes associated with the $\mathrm{Fe}$ core are confined to much lower frequencies, well below the range observed here. The qualitative agreement between the measured photodissociation spectra and the spectrum calculated for the model cluster raises the possibility that the spectra could be used to identify particular cluster geometries. It would be interesting to focus on individual cluster sizes and to seek quantitative agreement between theory and experiment. This approach may offer the best opportunity for determining the structures of these complexes.

Note added in proof. While examining the galley proofs, we discovered that the TOF spectra in Fig. 1 were recorded using $\mathrm{D}_{2}$, not with $\mathrm{H}_{2}$ as was stated in the text. However, the TOF spectra obtained with $\mathrm{H}_{2}$ were qualitatively similar to those shown, and this error does not affect any of the subsequent results or discussion.

\section{ACKNOWLEDGMENTS}

This work is supported by the U.S. Department of Energy, Office of Basic Energy Sciences, Division of Chemical Sciences, under Contract No. W-31-109-ENG-38 (M.B.K., G.M.K.); by National Science Foundation Grants Nos. DMRRUI 949085 (K.A.J.), NSF-DAAD INT-9514714 (M.R.P. and K.A.J.) and ONR Grant No. N0001498WX20709 (M.R.P.).
${ }^{1}$ M. E. Geusic, M. D. Morse, S. C. O'Brien, and R. E. Smalley, Rev. Sci. Instrum. 56, 2123 (1985).

${ }^{2}$ M. D. Morse, M. E. Geusic, J. R. Heath, and R. E. Smalley, J. Chem. Phys. 83, 2293 (1985).

${ }^{3}$ M. E. Geusic, M. D. Morse, and R. E. Smalley, J. Chem. Phys. 82, 590 (1985).

${ }^{4}$ R. L. Whetten, M. R. Zakin, D. M. Cox, D. J. Trevor, and A. Kaldor, J. Chem. Phys. 85, 1697 (1986).

${ }^{5}$ R. L. Whetten, D. M. Cox, D. J. Trevor, and A. Kaldor, Phys. Rev. Lett. 54, 1494 (1985)

${ }^{6}$ S. C. Richtsmeier, E. K. Parks, K. Liu, L. G. Pobo, and S. J. Riley, J. Chem. Phys. 82, 3659 (1985).

${ }^{7}$ E. K. Parks, K. Liu, S. C. Richtsmeier, L. G. Pobo, and S. J. Riley, J. Chem. Phys. 82, 5470 (1985).

${ }^{8}$ K. Liu, E. K. Parks, S. C. Richtsmeier, L. G. Pobo, and S. J. Riley, J. Chem. Phys. 83, 2882 (1985).

${ }^{9}$ W. F. Hoffman III, E. K. Parks, G. C. Nieman, L. G. Pobo, and S. J. Riley, Z. Phys. D 7, 83 (1987).

${ }^{10}$ E. K. Parks, B. H. Weiller, P. S. Bechthold, W. F. Hoffman, G. C. Nieman, L. G. Pobo, and S. J. Riley, J. Chem. Phys. 88, 1622 (1988).

${ }^{11}$ L. Zhu, J. Ho, E. K. Parks, and S. J. Riley, J. Chem. Phys. 98, 2798 (1993).

${ }^{12}$ L. Zhu, J. Ho, E. K. Parks, and S. J. Riley, Z. Phys. D 26, 313 (1993).

${ }^{13}$ A. Kaldor and D. M. Cox, J. Chem. Soc. Faraday Trans. 86, 2459 (1990).

${ }^{14}$ M. R. Zakin, R. O. Brickman, D. M. Cox, and A. Kaldor, J. Chem. Phys. 88, 3555 (1988).

${ }^{15}$ M. R. Zakin, D. M. Cox, and A. Kaldor, J. Chem. Phys. 89, 1201 (1988).

${ }^{16}$ A. Bérces, P. A. Hackett, L. Lian, S. A. Mitchell, and D. M. Rayner, J. Chem. Phys. 108, 5476 (1998).

${ }^{17}$ J. Conceicao, R. T. Laaksonen, L.-S. Wang, T. Guo, P. Nordlander, and R. E. Smalley, Phys. Rev. B 51, 4668 (1995).

${ }^{18}$ J. L. Whitten and H. Yang, Surf. Sci. Rep. 218, 55 (1996).

${ }^{19}$ J. Jellinek and Z. B. Güvenç, Physics and Chemistry of Finite Systems: From Clusters to Crystals (Kluwer, Richmond, VA, 1992), p. 1047.

${ }^{20}$ J. Jellinek and Z. B. Güvenç, Z. Phys. D 19, 371 (1991).

${ }^{21}$ J. Jellinek and Z. B. Güvenç, Nuclear Physics Concepts in the Study of Atomic Cluster Physics (Lecture Notes in Physics 404) (Springer, Bad Honnef, Germany, 1991).

${ }^{22}$ Y. L. Alvarez, G. E. López, and A. J. Cruz, J. Chem. Phys. 107, 1420 (1997)

${ }^{23}$ K. Christmann, Surf. Sci. Rep. 9, 1 (1988).

${ }^{24}$ G. Dietrich, K. Dasgupta, K. Lützenkirchen, L. Schweikard, and J. Ziegler, Chem. Phys. Lett. 252, 141 (1996).

${ }^{25}$ M. B. Knickelbein, J. Chem. Phys. 104, 3517 (1996).

${ }^{26}$ E. K. Parks, T. D. Klots, and S. J. Riley, J. Chem. Phys. 92, 3813 (1990).

${ }^{27}$ M. B. Knickelbein and W. J. C. Menezes, J. Chem. Phys. 94, 4111 (1991).

${ }^{28}$ M. B. Knickelbein and W. J. C. Menezes, Chem. Phys. Lett. 184, 433 (1991).

${ }^{29}$ M. B. Knickelbein and W. J. C. Menezes, J. Phys. Chem. 96, 6611 (1992).

${ }^{30}$ M. R. Zakin, D. M. Cox, R. L. Whetten, D. J. Trevor, and A. Kaldor, Chem. Phys. Lett. 135, 223 (1987).

${ }^{31}$ R. Smoluchowki, Phys. Rev. 60, 661 (1941).

${ }^{32}$ E. K. Parks, G. C. Nieman, L. G. Pobo, and S. J. Riley, J. Phys. Chem. 91, 2671 (1987).

${ }^{33}$ S. Yang and M. B. Knickelbein, J. Chem. Phys. 93, 1533 (1990).

${ }^{34}$ B. A. Collings, K. Athenassenas, D. M. Rayner, and P. A. Hackett, Chem. Phys. Lett. 227, 490 (1994).

${ }^{35}$ M. B. Knickelbein, J. Chem. Phys. 104, 3517 (1996).

${ }^{36}$ A. M. Baró and W. Erley, Surf. Sci. 112, 759 (1981).

${ }^{37}$ P. Hohenberg and W. Kohn, Phys. Rev. 136, 864 (1964).

${ }^{38}$ W. Kohn and L. J. Sham, Phys. Rev. A 140, 1133 (1965).

${ }^{39}$ R. O. Jones and O. Gunnarson, Rev. Mod. Phys. 61, 689 (1989).

${ }^{40}$ D. C. Patton, D. V. Porezag, and M. R. Pederson, Phys. Rev. B 55, 7454 (1997).

${ }^{41}$ J. L. Chen, C. S. Wang, K. A. Jackson, and M. R. Pederson, Phys. Rev. B 44, 6558 (1991)

${ }^{42}$ M. Castro and D. R. Salahub, Phys. Rev. B 47, 10955 (1993).

${ }^{43}$ P. Ballone and R. O. Jones, Chem. Phys. Lett. 233, 632 (1995).

${ }^{44}$ M. Castro, C. Jamorsky, and D. R. Salahub, Chem. Phys. Lett. 271, 133 (1997).

${ }^{45}$ B. J. Dunlap, Phys. Rev. A 41, 5691 (1990).

${ }^{46}$ K. A. Jackson and M. R. Pederson, Phys. Rev. B 42, 3276 (1990).

${ }^{47}$ M. R. Pederson and K. A. Jackson, Phys. Rev. B 41, 7453 (1990).

${ }^{48}$ D. V. Porezag and M. A. Pederson, Phys. Rev. B 54, 7830 (1996). 